\title{
MR perfusion imaging: correlation with PET and quantitative angiography
}

\author{
J. Schwitter, G.K. von Schulthess * \\ MR Centre. University Hospital Zurich, Ramistr. 100, Zurich CH-8091, Switzerland
}

Received 4 August 2000; accepted 4 August 2000

Keywords: Cardiac; MRI; Angiography

\section{Background}

Monitoring contrast medium $(\mathrm{CM})$ wash-in kinetics in dipyridamole induced hyperemia of the myocardium by MR allows detection of stenosed coronary arteries. In this study, the diagnostic performance of signal intensity (SI) upslope data as obtained from MR data sets was determined and compared with $\mathrm{N}-13$ ammonia rest and dipyridamole stress $P E T$ and quantitative coronary angiography (QCA).

\section{Methods}

Forty-seven patients and 18 healthy subjects were studied by MR using a hybrid echo-planar pulse sequence (1.5 T cardiac optimised scanner $\mathrm{GE}$, Waukesha, USA) for monitoring myocardial first pass kinetics of Gd-DTPA-BMA (Omniscan ${ }^{\mathrm{TM}}$, Nycomed Imaging SA, Norway; $0.1 \mathrm{mM} / \mathrm{kg}$ injected at $3 \mathrm{ml} / \mathrm{s} \mathrm{IV)}$ during hyperemia (dipyridamole $0.56 \mathrm{mg} / \mathrm{kg}$ ). The technique is able to measure at least four myocardial slices during the passage of a single bolus, thereby covering most of the relevant myocardial regions. SI upslope as a measure of myocardial perfusion was calculated from pixelwise parametric maps in the subendocardial layer and for full wall thickness in 32 sectors per heart or eight sectors per slice. In addition, quantitative tools for obtaining pixelwise slope maps of registered MR perfusion data sets were developed and bull's eye plots of MR slope map data were generated. Prior to coronary angiography, coronary flow reserve (CFR; hyperemial

\footnotetext{
* Corresponding author.

E-muil address: vonschulthess $(u d$ dmr.usz.ch (G.K. von Schulthess).
}

induced by dipyridamole $0.56 \mathrm{mg} / \mathrm{kg}$ ) was determined in corresponding sectors by ${ }^{13} \mathrm{~N}$-ammonia PET [GE Advance, (Walukesha. USA) and N-13 ammonia produced in-house on a PET-TRACE 2000 cyclotron (Uppsala, Sweden) at resting conditions and during hyperemia, as PET is considered to be the gold standard for myocardial perfusion imaging. Receiver operator characteristics (ROC) of subendocardial upslope data for the detection of coronary artery disease were generated and analysed.

\section{Results}

Consistent signal enhancement during perfusion of around $250-300 \%$ were obtained using the pulse sequence. This resulted in stable and easy to interpret data sets. (ROC) of subendocardial upslope data for the detection of coronary artery disease (defined by PET as mean CFR-2SD of controls) show a sensitivity and specificity of 91 and $94 \%$, respectively, with an area under the ROC curve (AUC) of 0.93. In comparison with QCA (diameter stenosis $\geq 50 \%$ ), sensitivity and specificity was 87 and $85 \%$ (AUC of 0.91 ), respectively, for $M R$, and 91 and $81 \%$ (AUC of 0.93 ), respectively. for PET. Direct comparison of upslope data and myocardial blood flow determined by PET revealed an underestimation of high flows by MR.

\section{Conclusions}

The presented MR approach reliably identifies pattients with anatomically and hemodynamically signifcant coronary artery stenoses. This is due to the fact. 
that the pulse sequence used produces a substantial change in signal intensity in the perfused versus poorly perfused myocardial regions. Analysis of upslope in this setting rather than of other parameters provides a very sensitive and specific measure of myocardial ischemia. As upslope is a semiquantitative measure of absolute perfusion, even patients with triple vessel disease may be evaluated using this method. This is not the case when using conventional nuciear techniques. Further- more, the spatial resolution of the MR images permits one to resolve the subendocardial layers of the myocardium, which thus can be evaluated separately from the entire wall. Again, this is not possible using nuclear cardiology perfusion imaging. The robustness of this MR perfusion imaging approach and the fact, that most of the heart can be covered may qualify for its clinical application in the management of coronary artery disease. 